$\langle(\bullet\rangle)$ Base Station
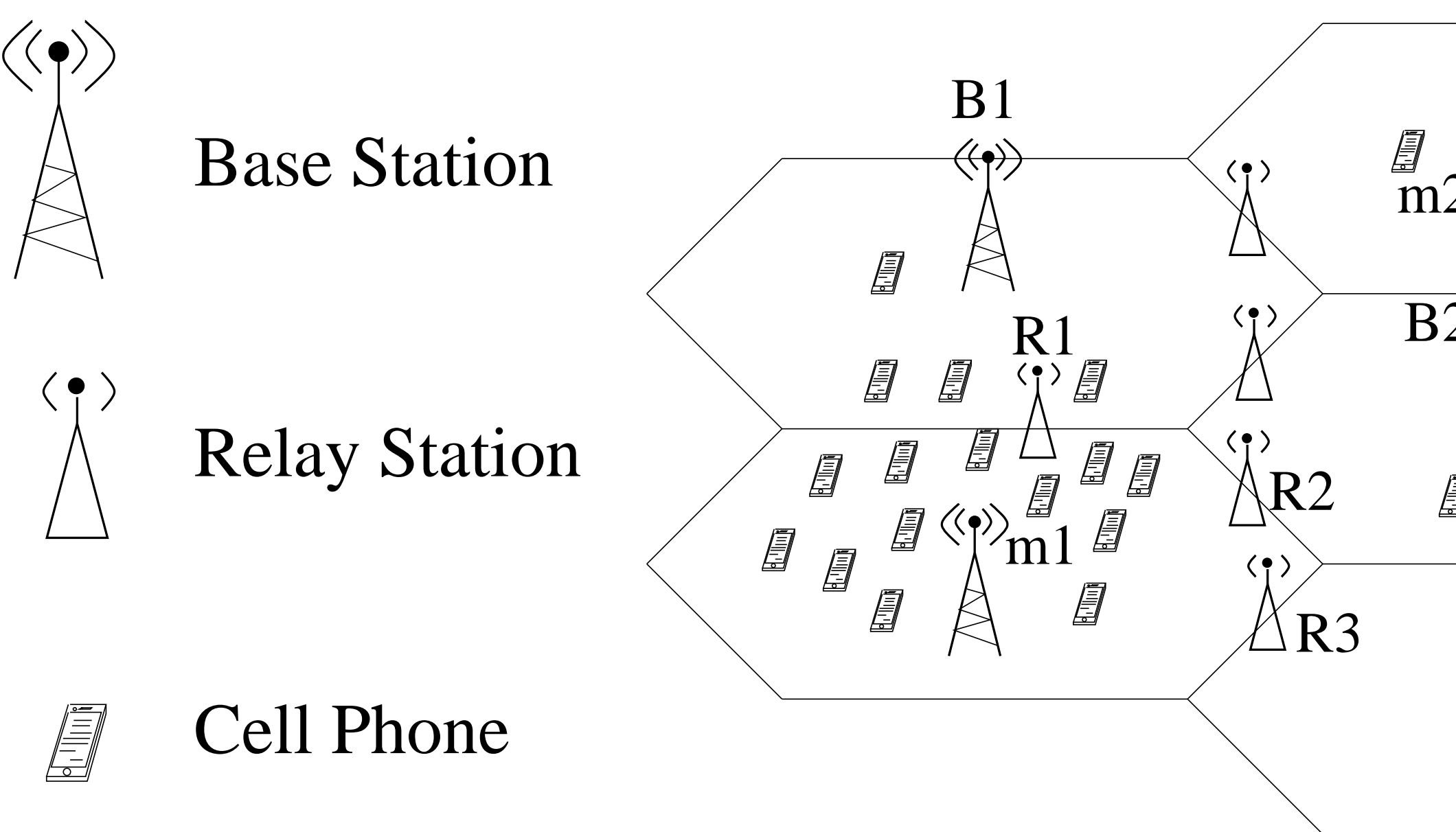
$\langle(\varphi)\rangle \quad$ Base Station

$\bigwedge^{\langle\dot{j}}$ Relay Station

息 Cell Phone 
$\langle(\bullet\rangle)$ Base Station

$\mathfrak{j}^{\rangle}$Relay Station

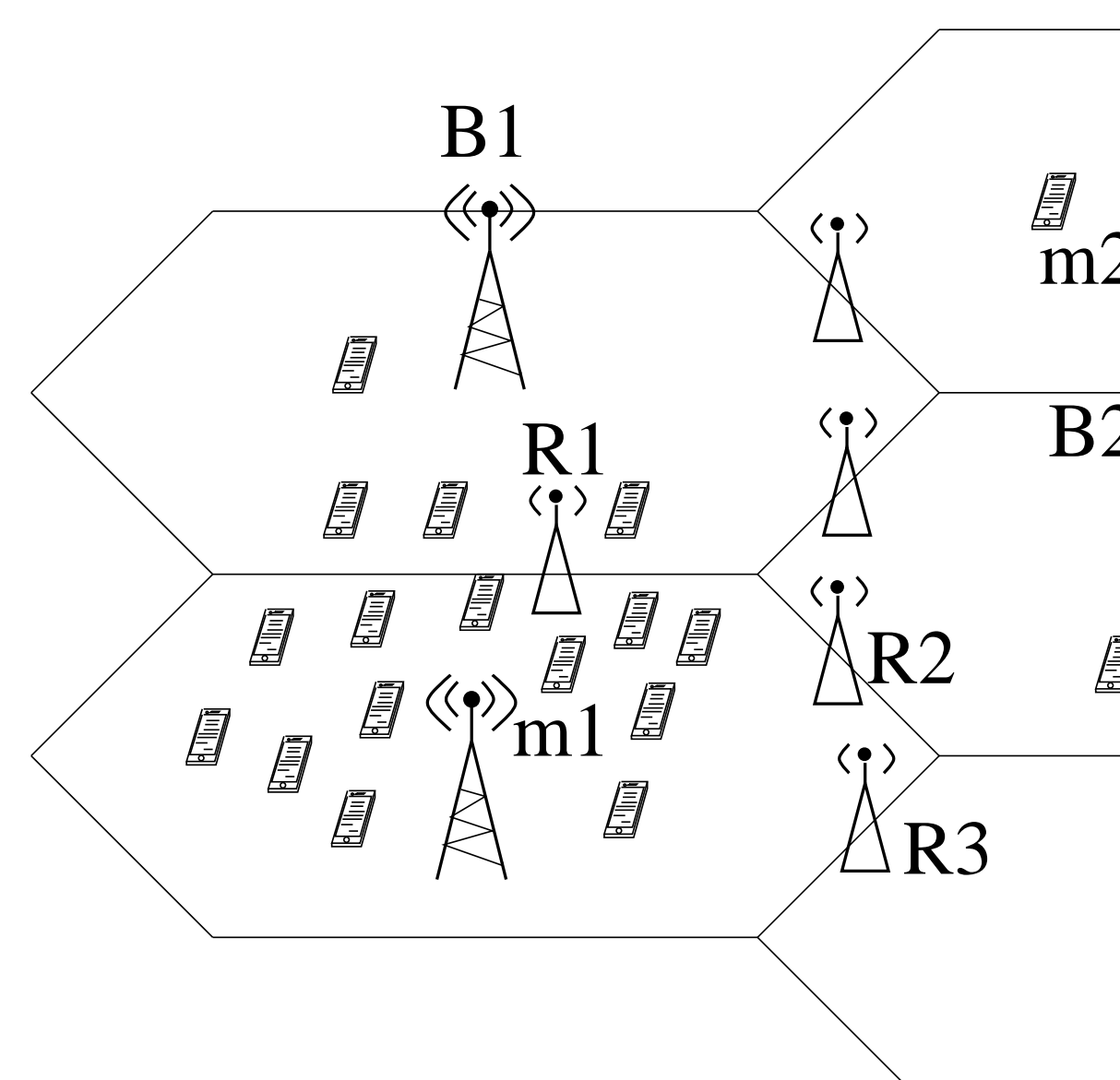




\section{Location Aided Energy Balancing Strategy in Green Cellular Networks}

\author{
Jianjun Yang \\ Department of Computer Science \\ University of North Georgia \\ Email: jianjun.yang@ung.edu \\ Zongming Fei \\ Lab for Advanced Networking \\ Department of Computer Science \\ University of Kentucky \\ Email: fei@netlab.uky.edu
}

\author{
Bryson Payne \\ Department of Computer Science \\ University of North Georgia, USA \\ Email: bryson.payne@ung.edu
}

\author{
Le $\mathrm{Li}$ \\ David R.heriton School \\ of Computer Science \\ University of Waterloo, Canada \\ Email: 1248li@uwaterloo.ca
}

\author{
Markus Hitz \\ Department of Computer Science \\ University of North Georgia, USA \\ Email: markus.hitz@ung.edu \\ Tongquan Wei \\ School of Information Science \\ and Technology \\ East China Normal University, China \\ Email: tqwei@cs.ecnu.edu.cn
}

\begin{abstract}
Most cellular network communication strategies are focused on data traffic scenarios rather than energy balance and efficient utilization. Thus mobile users(cell phones) in hot cells may suffer from low throughput due to energy loading imbalance problem. In state-of-art cellular network technologies, relay stations extend cell coverage and enhance signal strength for mobile users. However, busy traffic makes the relay stations in hot area run out of energy quickly. In this paper, we propose an energy balancing strategy in which the mobile nodes are able to dynamically select and hand over to the relay station with the highest potential energy capacity to resume communication. Key to the strategy is that each relay station merely maintains two parameters that contains the trend of its previous energy consumption and then predicts its future quantity of energy, which is defined as the relay station's potential energy capacity. Then each mobile node can select the relay station with the highest potential energy capacity. Simulations demonstrate that our approach significantly increase the aggregate throughput and the average life time of relay stations in cellular network environment.
\end{abstract}

\section{INTRODUCTION}

During the past several years, there has been tremendous growth in cellular networks. With the introduction of Android and iPhone devices, use of ebook readers such as iPad and Kindle and the success of social networking giants such as Facebook, WeChat and QQ, the number of customers and the demand for cellular traffic have escalated significantly. The huge amount of customers and very high volume data transmission result in serious problems of energy consumption[1].

The rising energy costs of operating cellular networks have led to an emerging trend of addressing energy efficiency and energy balance utilization amongst the network operators and regulatory bodies such as 3GPP and ITU[2]. This trend has stimulated the interest of researchers in an innovative new research area called green cellular networks. In this regard, the European Commission has recently started new projects to address the energy issue of mobile communication systems, such as "Energy Aware Radio and Network Technologies (EARTH)", "Towards Real Energy-efficient Network Design" and "Cognitive Radio and Cooperative strategies for Power
Saving in Multi-standard Wireless Devices" [3]. Energy in cellular network is a vast research discipline that needs to cover all the layers of the protocol stack and various system architectures and it is important to identify the fundamental trade-offs linked with energy efficiency and the overall performance [4].

Scholars have addressed four key issues in terms of energy efficiency with network performance[2]. They are deployment efficiency (balancing deployment cost, throughput), spectrum efficiency (balancing achievable rate), bandwidth (balancing the bandwidth utilized) and delay (balancing average endto-end service delay). To address the challenge of increasing power efficiency in future cellular networks and thereby to maintain profitability, it is crucial to consider various paradigm-shifting technologies, such as energy efficient wireless architectures and protocols, efficient base station(BS) redesign, smart grids, opportunistic network access or cognitive radio, cooperative relaying and heterogeneous network deployment based on smaller cells.

In addition to academia, governments and industries have recently shown keen concerns on the critical issues related to energy efficiency and its balance utilization and in the ICT(Information and Communication Technology) area. However, as studied in the recent literature [5][6], most of the techniques applied to current mobile networks have been designed by taking into account non-energy-related factors, such as throughput, Quality of Service (QoS), availability, scalability, and so on. Moreover, in real-world systems, mobile users (MUs) are not evenly distributed across cells, resulting in that MUs in a hot cell will be affected by the load imbalance and they might unable to get services. Meanwhile, as novel network architectures that include picocells, hierarchical cells and femtocells emerge, the density of base stations and mobile users is becoming larger, and the cells go smaller. The appearance of this high density cellular networks introduces more variety of load across different cells and makes the load imbalance problem more serious.

The new generation cellular networks allow mobile users to connect relay station and then connect to base station. The busy traffic near a relay station often makes the energy of the 
relay station go down very quickly. However, the relay station with low traffic is idle with high energy. In order to balance the energy utilization among different cells, it is needed to transfer the over-loaded traffic from hot cells to neighboring cooler ones. The challenge is how to balance the energy utilization of the relay stations in order to get the best trade-off among all the relay stations in a cellular network.

In this paper, we develop a statistical parameter based energy balance utilization algorithm, in which each relay station maintains the acceleration and the variance of energy consumption that represent the station's historical energy consumption acceleration and the variance. Together with its current energy quantity, the relay station is able to predict its future energy quantity, which is considered as its potential energy capacity. Before payload data transmission between a mobile user and a relay station, the mobile user disseminates a message to a portion of its neighbor relay stations depending on their geographic locations. Then it selects and hands over to the relay station with highest potential energy. Because each relay station only needs to maintain two parameters, the overhead of this scheme is very low. Our simulation results illustrate that our approach significantly increases the aggregate throughput in the network and the average life time of the relay stations compared with existing approaches.

The rest of the paper is organized as follows. Section II discusses the related research on this topic. Section III proposes a novel method that select the best relay station. We evaluate the proposed schemes by simulations and describe the performance results in Section IV. Section V concludes the paper.

\section{RELATED WORK}

Various energy utilization strategies in cellular networks were proposed. The schemes are classified into several categories. The first one contains the strategies based on channel borrowing from cooler cells[9]. The second category includes the strategies based on BS selection [10]. The third category contains strategies based on power control and cell breathing[10][11]. The last type consists of strategies on relayassisted traffic transfer[12][13][14]. The basic idea of channel borrowing is to borrow a set of channels from cooler cells to hot cells. However, this will change the pre-defined spectrum reuse pattern and introduce more co-channel interference[7]. There are also a great deal of research combining multihop wireless network (MWN) with infrastructure wireless networks, forming architecture of multi-hop cellular network (MCN). In MCNs, relay stations (RSs) are network components that are dedicated to storing and forwarding data received from BSs to MUs, and vice versa. Deploying relay stations can extend the coverage and enhance the signal strength, which clearly help improve the performance for MUs near the edge of the cell. The appearance of MCNs also provides another method to solve the load imbalance among different cells. That is to transfer over-loaded traffic from hot cells to cooler cells by RSs. Compared with previous discussed dynamic load balancing schemes, relay-based load balancing schemes are more flexible and will introduce less interference. In [5], a mobile-assisted call admission scheme is proposed to achieve load balancing in cellular networks, which require an ad-hoc overlay network on the cellular network. The authors divided the channels into two parts, one for the ad-hoc overlay network, the other for the cellular network. In [6][7], the authors proposed dynamic load balancing schemes in the integrated cellular and ad-hoc relaying systems (iCAR). The ad-hoc relaying stations (ARS) compose an overlay ad-hoc network, which can help relay traffic among different cells. As in iCAR systems, ARSs work on ISM-band channels, it is pointed out that the performance of iCAR systems will depend on the number of available ISM-band channels, and interference in ISM-band could affect the performance of dynamic load balancing.

Z. Yang and Z. Niu[15] proposed a relay-based load balancing scheme, in which no fixed division of channels is made and all transmissions work on the same spectrum. According to an association control algorithm, MUs and RSs re-associate from hot cells to neighboring cooler cells to balance the load. This scheme improves performance of boundary MUs.

A. Alam et al. [17] investigates dynamic traffic-aware BS switching modes, where the BS can alter its operating modes between standard BS operations and switching to relay station (RS) mode, the so-called BS-RS Switching model. Depending on the traffic fluctuations, load profiles are divided into two categories. One is Zero-to-medium traffic period when a BS switches to the RS mode and turns off all its high-power consuming equipment. The second is Peak traffic period when all BS are fully active. The rationale for switching from a BS to RS mode is to ensure those MS that would be served by the switched off cell and may suffer deep fading, are still be able to receive the same QoS. Furthermore, since the propagation distance has been shortened between the MS and serving BS via the back haul connection, the required MS transmit power is concomitantly reduced compared with the BS sleep and cell zooming technique [5].

K. $\mathrm{Xu}$ and $\mathrm{M}$. Zhou [18][19] developed a realistic and representative energy model based on RF transceiver. As a typical IEEE 802.15.4 RF transceiver mode, each wireless node is equipped with multiple discrete transmit power levels. Based on this energy model, they developed a power control policy, which configure the transmit power level as a function of transmission distance such that the energy cost is minimized. Then, the optimized energy balanced chain model is proposed to determine the optimal traffic flow distribution.

Y. Gu and M. Pan [21][22] studied how to maximize the life time of delay-sensitive WSNs via joint routing and sleep scheduling. They have mathematically formulated the lifetime maximization problem under energy provision, routing, sleep scheduling, and delay constraints into a MINLP problem, among which energy is essential to determine the life time of wireless devices.

\section{PROBLEM Formulation}

\section{A. The Basic Idea}

In cellular networks, each base station covers a number of cell phones in a hexagonal cell. We consider a cellular network containing a set of base stations(BS), relay stations(RS) and mobile users(cell phones)(MU), denoted by $\left\{B_{1}, B_{2}, \ldots, B_{s}\right\}$, $\left\{R_{1}, R_{2}, \ldots, B_{t}\right\}$, and $\left\{m_{1}, m_{2}, \ldots, m_{p}\right\}$. Each BS is located at the center of the cell. Each RS is located at a boundary 
of cells. The traditional communication between two mobile users $m_{i}$ to $m_{j}$ is that $m_{i}$ communicates to its base station $B_{i}$, then $B_{i}$ communicates to $B_{j}$ which covers $m_{j}$, and then $B_{j}$ communicates to $m_{j}$. In new generation cellular network, the relay stations are used to assist communications between cell phone to cell phone or cell phone to base station. Fig. 1 shows an instance with several base stations, relay stations and cell phones, where the central cell is a hot cell with a large number of cell phones and busy data traffic and the other cells are cooler cells with less cell phones. Note that the cell phones in all figures of this paper represent active cell phones.

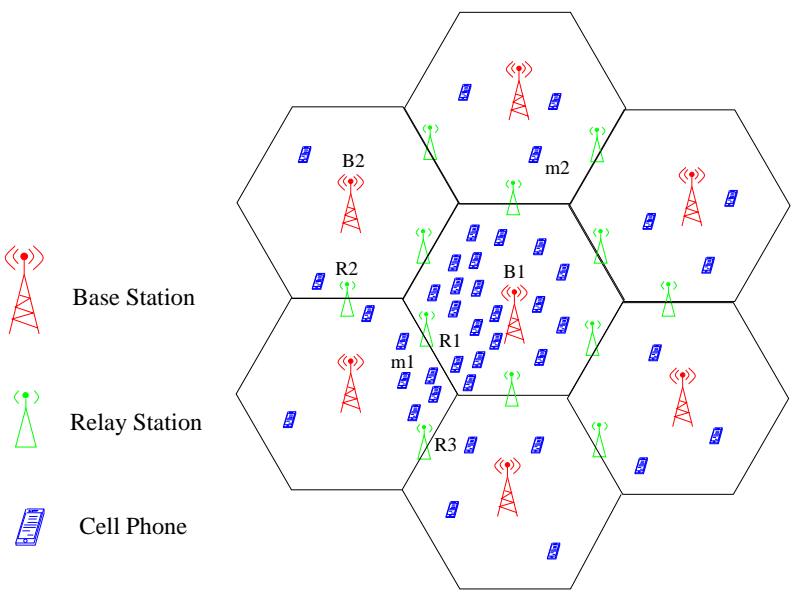

Fig. 1: A topology of a cellular network.

Data transmission takes place when a cell phone(initiator) intends to talk to another cellphone(receiver). The scenario is simple if the two are in the same cell[8]. We consider the circumstance that the receiver is in another cell. In Fig. 1, the communication between $m_{1}$ and $m_{2}$ is such an instance, in which data is relayed from $m_{1}$ to $R_{1}$, then from $R_{1}$ to $B_{1}$, and continues until it reaches $m_{2}$. In the scenario, $R_{1}$ is hot with a lot of cell phones nearby. Hence its energy is going down very quickly. However, $R_{2}$ covers less cell phones and its energy is going down slowly. Here comes up with a question, can $m_{1}$ find $R_{2}$ or even $R_{3}$ to relay the message to bypass the hot relay station $R_{1}$ ?

Since a mobile cell phone is able to select a relay station and hand over to it[8], we develop an approach to let each mobile user find the RS with highest potential energy and hand over to it. We start our basic idea by a simplified case as Fig. 2. Suppose $m_{1}$ intends to talk to $m_{2}$. If $m_{1}$ selects $R_{1}$ to conduct relay, it may not be a good choice because $R_{1}$ is busy and may run out of energy shortly. $R_{1}, R_{2}$ and $R_{3}$ are all possible relay stations for $m_{1}$. Suppose the residual energy of $R_{1}$ is $900 \mathrm{kWh}, R_{2}$ is $800 \mathrm{kWh}$ and $R_{3}$ is $850 \mathrm{kWh}$ at current moment. We further assume that the historical residual energy vector up to this moment of $R_{1}$ is $[\ldots, 2000,1500$, $1300,900], R_{2}$ is [.., 900, 870,830,800], and $R_{3}$ is [.., 1400, $1200,1000,850]$. A simple strategy will let $m_{1}$ select the one that has the highest energy at the moment. Seemingly, $R_{1}$ is the best choice. However, since $R_{1}$ is in a hot area serving a large number of mobile users and its energy is going down dramatically, while $R_{2}$ are serving much less mobile users and its energy goes down slowly, $m_{1}$ should select $R_{2}$.
Our goal is to let $m_{1}$ select the relay station that will have the highest potential energy. In our approach, each RS can predict its future energy right after this time slot based on its historical and current energy, which is defined as potential energy of the RS in this paper. In this example, our strategy will let $m_{1}$ choose $R_{2}$.

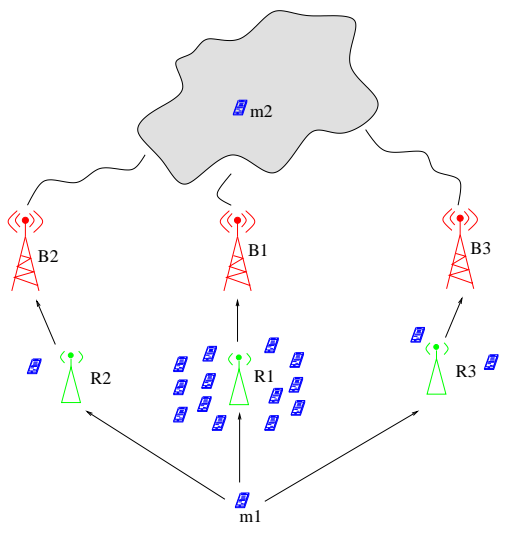

Fig. 2: The scenario to illustrate the main idea.

\section{B. Computation of Potential Energy}

The mobile initiator needs to know which relay station is the one that has the potential highest energy. The energy varying trend is essential to determine an RS's potential energy. Suppose the current time is $k$. A naive method on predicting the energy at time $k+1$ is as follows. Let $\mathrm{RS} R_{i}$ save all its historical and current (time $k$ ) energy $E_{i_{0}}, E_{i_{1}}, \ldots, E_{i_{k}}$ in a vector. Then it can predict its energy for time $k+1$ by curve-fitting in numerical analysis that approximates its moving trend. The limitations of this method are to spend too much space to save the energy values in the vector and too complex for curve-fitting computation.

We propose a statistical based strategy to let each relay station predict its future energy. In this scheme, each RS only needs to maintain two parameters. One is its potential acceleration and the second is the variance of the acceleration. The two parameters store how its residual energy changes. Together with the RS's current energy, its future energy capacity can be calculated and is considered its potential energy. Thus, mobile user $m_{1}$ is able to figure out the best RS subsequently.

We define the following notations to represent the terms regarding our approach for node $R_{i}$.

$E_{i_{k}}$ : The measured energy at time $k$.

$\hat{E_{i_{k}}}$ : The energy predicted at time $k$.

$a_{i_{k}}$ : The acceleration measured at time $k$. It indicates how energy changes during a time slot.

$\hat{a_{i_{k}}^{-}}$: The acceleration at time $k$ evolved from time $k-1$.

$\hat{a_{i}}$ : The potential acceleration at time $k$.

$v_{i_{k}}$ : The variance of acceleration updated at time $k$.

$v_{i_{k}}^{-}$: The variance of acceleration at time $k$ evolved from time $k-1$.

$\epsilon$ : The error or noise in the process.

$B_{i_{k}}$ : The blending factor at time $k$. 
At time $k, R_{i}$ measures its energy $E_{i_{k}}$. And then it computes $a_{i_{k}}$ as its measured acceleration by

$$
a_{i_{k}}=\left(E_{i_{k}}-E_{i_{k-1}}\right) / \Delta t
$$

$R_{i}$ updates $\hat{a_{i_{k}}^{-}}$and $v_{i_{k}}^{-}$in order to keep its historical energy to predict its future energy.

$$
\begin{gathered}
\hat{a_{i_{k}}^{-}}=a_{i_{k-1}} \\
v_{i_{k}}^{-}=v_{i_{k}-1}+\epsilon
\end{gathered}
$$

$R_{i}$ also computes the blending factor $B_{i_{k}}$, which indicates how much the acceleration changes from last time to current time.

$$
B_{i_{k}}=v_{i_{k}}^{-}\left(v_{i_{k}}^{-}+\epsilon\right)^{-1}=v_{i_{k}}^{-} /\left(v_{i_{k}}^{-}+\epsilon\right)
$$

Once $R_{i}$ obtains the blending factor $B_{i_{k}}$ and the evolved acceleration $\hat{a_{i_{k}}^{-}}$, it knows how much the acceleration changes and the evolved acceleration. Additionally, $R_{i}$ considers the measured acceleration $a_{i_{k}}$. Then it calculates its potential acceleration $\hat{a_{i_{k}}}$. This acceleration will be used to predict its energy of time $k+1$.

$$
\hat{a_{i_{k}}}=\hat{a_{i_{k}}^{-}}+B_{i_{k}}\left(a_{i_{k}}-\hat{a_{i_{k}}}\right)
$$

$R_{i}$ updates the variance of acceleration for future utilization.

$$
v_{i_{k}}=\left(1-B_{i_{k}}\right) v_{i_{k}}^{-}
$$

Finally, the energy predicted of time $k+1$ is

$$
E_{i_{k+1}}=E_{i_{k}}+\hat{a_{i_{k}}} \Delta t
$$

At a certain time $k, R_{i}$ only needs to measure its energy $E_{i_{k}}$ and record two parameters $\hat{a_{i_{k-1}}}$ and $v_{i_{k-1}}$. Then it can predict its energy at time $k+1$ by the calculation, which is the potential energy capacity of $R_{i}$.

\section{Algorithm Description}

In our approach, when a user $m_{1}$ intends to communicate to $m_{2}$, it selects the relay station with highest potential energy. Apparently, $m_{1}$ does not need to consider the relay stations in the opposite direction $m_{1} \rightarrow m_{2}$, such as $R_{4}, R_{5}$ and $R_{6}$ in Fig. 3. Here $R_{1}, R_{2}$ and $R_{3}$ are candidates for $m_{1}$. How can $m_{1}$ know the three possible candidates among the six relay stations in the boundaries? In new generation cellular networks, each device is equipped with GPS and hence it knows its location. We assume that the initiator $m_{1}$ knows the location of the receiver $m_{2}$. The assumption is very common in geographic routing[20]. So $m_{1}$ finds out the three possible candidates in this way: It connects $m_{1}$ and $m_{2}$. The line segment intersects the hexagonal area at $p$. Then the relay station located at the edge where $p$ is located and the two relay stations located at its adjacent edges are considered as good relay candidates. In Fig. 3, $p$ is located at segment $b c$, so $R_{2}$ located at $b c$ is considered as one of the candidates. The two adjacent edges of $b c$ are $a b$ and $c d$, so $R_{1}$ and $R_{3}$ are also considered as $m_{1}$ 's candidates.

In our strategy, when a cell phone(initiator) intends to talk to a receiver in a different cell, it calls the procedure

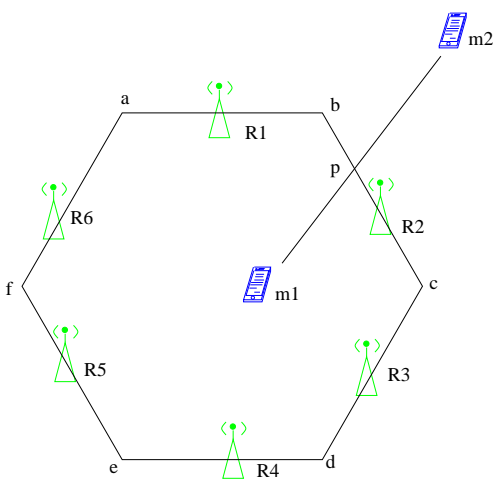

Fig. 3: To figure out three good relay stations.

Detection() to figure out the three possible relay stations by its location and the destination's location. In the procedure, it sends out a detection message to the three relay stations. Upon receiving the detection message, each RS calls Prediction() to calculate its potential energy and inform the energy capacity to the initiator. The initiator calls Selection() to find the relay station with possible highest potential energy, hands over to it and sends its payload to the RS.

\section{Detection()}

Figure out the three candidate relay stations by its location and destination's location

Send detection message to the three relay stations

Fig. 4: Algorithm for initiator(MU) to send detection message

\section{Prediction()}

Calculate its potential energy by formula (7)

Send the energy quantity back to the initiator

Fig. 5: Algorithm of computation for potential energy

\section{Selection()}

Compare the three energy quantities from the three relay stations Select the RS with highest energy values

Hands over to the RS

Send payload to the RS

Fig. 6: Algorithm of hand over

\section{PERFormance EVAluation}

The performance evaluation was conducted in a simulated noiseless radio network environment using MATLAB. We create a topology that consists of 20 hexagonal cells. A base station is located in the center of each cell and a relay station is located at each edge. We randomly distribute a number of mobile users to the cells. We performed a sequence of experiments in which the number of mobile users is changed from 200 to 800 with increment of 50. For each number of mobile users, we measure the aggregate throughput 10 times and present the average. We also measure the average life time of the relay stations in our experiments. The life time of a relay station is important. If one RS runs out of energy and turns 
down, there will be a hole[16] and the whole throughput will be decreased dramatically.

Our approach considers energy balance. We compare our approach("EB by MU") with two other approaches. One is the scheme which considers energy balance but the balance is determined by base station("EB by BS"). The second one is without considering energy balance("No EB").

Fig. 7 shows our evaluation of aggregate throughput. Among the three approaches, the scheme without energy balance("No EB") has the lowest throughput. This indicates that energy balance can improve aggregate throughput because the traffic are allocated more reasonable to the relay stations. The throughput of our method is the highest one.

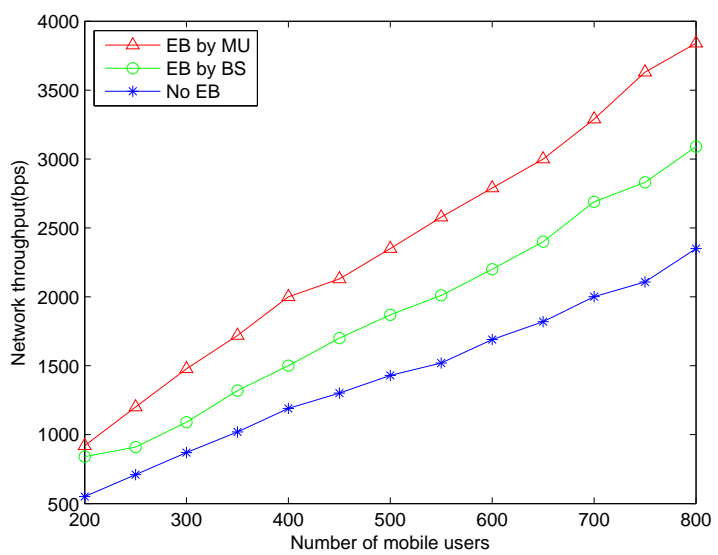

Fig. 7: Aggregate throughput

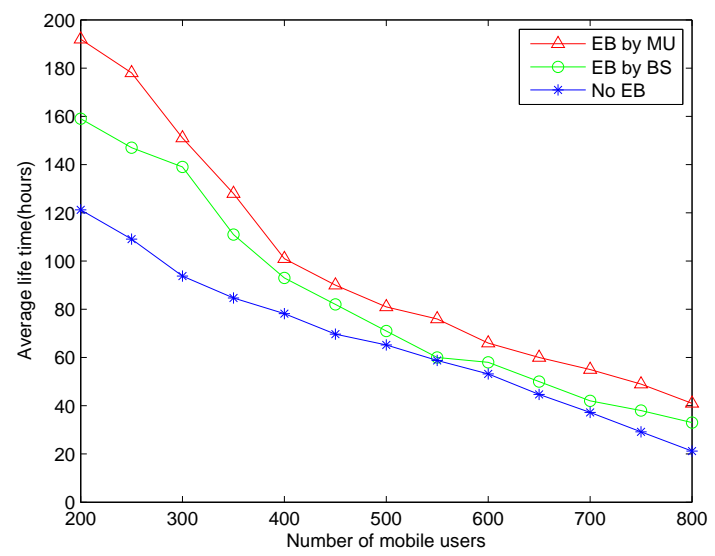

Fig. 8: Life time of relay stations

Fig. 8 shows the life time of the three schemes. The one without energy balance("No EB") results in the lowest average lift time. That is because when a relay station has busy traffic load, it runs of out energy quickly. And then there is a void or hole. The regular solution for hole problem is to find the landmark relay station [16]. However, the landmark has very heavy burden and it is exhausted quickly. This makes the network much worse. Our approach has the longest lift time because the communication between two cell phones is always relayed by the RS with the highest potential energy. If a RS is busy and its energy goes down significantly, the new communication will not rely on it. Hence the balanced energy utilization makes the life time longer.

\section{COnClusion}

In this paper, we present a statistical based mechanism to balance energy utilization in cellular network. In the approach, each relay station only needs to remain two factor to store its historical energy trend and then it is able to predict its future energy. The message initiator selects the relay station with potential highest energy to relay data transmission. Simulations demonstrate that our approach results in higher throughput in the network and longer average life time of relay stations over related approaches.

\section{REFERENCES}

[1] G. Fettweis and Z. Ernesto, ICT energy consumption-trends and challenges, Proceedings of the 11th International Symposium on Wireless Personal Multimedia Communications. Vol. 2. No. 4. 2008.

[2] H. Ziaul, H. Boostanimehr and V.K. Bhargava, Green cellular networks: A survey, some research issues and challenges, Communications Surveys \& Tutorials, IEEE 13.4 (2011): 524-540.

[3] M. Gruber, et al., EARTH: energy aware radio and network technologies, ersonal, Indoor and Mobile Radio Communications, 2009 IEEE 20th International Symposium on. IEEE, 2009.

[4] Y. Chen, et al., Fundamental trade-offs on green wireless networks,Communications Magazine, IEEE 49.6 (2011): 30-37.

[5] X. wang, et al., A survey of green mobile networks: Opportunities and challenges,obile Networks and Applications 17.1 (2012): 4-20.

[6] M. Etoh, T. Ohya and Y. Nakayama, Energy consumption issues on mobile network systems, Applications and the Internet, 2008. SAINT 2008. International Symposium on. IEEE, 2008.

[7] J. Yang and Z. Fei, Bipartite Graph Based Dynamic Spectrum Allocation for Wireless Mesh Networks, Distributed Computing Systems Workshops, 2008. ICDCS'08. 28th International Conference on (pp. 96-101). IEEE, Beijing, 2008 .

[8] G. Josh, On Relay-assisted Cellular Networks, thesis, Department of Electrical Engineering, Indian Institute of Technology-Mombay, June, 2010

[9] S. Patra, et al., Improved genetic algorithm for channel allocation with channel borrowing in mobile computing,Mobile Computing, IEEE Transactions on 5.7 (2006): 884-892.

[10] S. Hanly, An algorithm for combined cell-site selection and power control to maximize cellular spread spectrum capacity,Selected Areas in Communications, IEEE Journal on 13.7 (1995): 1332-1340.

[11] M. Soleimanipour, W. Zhuang and G.H. Freeman, Optimal resource management in wireless multimedia wideband CDMA systems, SMobile Computing, IEEE Transactions on 1.2 (2002): 143-160.

[12] X. Wu, B. Mukherjee and S. Chan, Maca-an efficient channel allocation scheme in cellular networks, Global Telecommunications Conference, 2000. GLOBECOM'00. IEEE. Vol. 3. IEEE, 2000.

[13] $\mathrm{H}$. Wu, et al., Integrated cellular and ad hoc relaying systems: iCAR, Selected Areas in Communications, IEEE Journal on 19.10 (2001): 21052115.

[14] E. Yanmaz, and O. Tonguz, Dynamic load balancing and sharing performance of integrated wireless networks, Selected Areas in Communications, IEEE Journal on 22.5 (2004): 862-872.

[15] Z. Yang, and Z. Niu, A new relay based dynamic load balancing scheme in cellular networks, Vehicular Technology Conference Fall (VTC 2010Fall), 2010 IEEE 72nd. IEEE, 2010.

[16] J. Yang, and Z. Fei, ITGR: Intermediate Target Based Geographic Routing, Computer Communications and Networks (ICCCN), 2010 Proceedings of 19th International Conference on (pp. 1-6). IEEE, Zurich, Switzerland, August 2010. 
[17] S. Alam, et al., Energy efficient relay-assisted cellular network model using base station Switching, Globecom Workshops (GC Wkshps), 2012 IEEE. IEEE, 2012.

[18] K. XU and M. Zhou, Energy balanced chain in IEEE 802.15. 4 low rate WPAN, Computing, Networking and Communications (ICNC), 2013 International Conference on. IEEE, 2013.

[19] K. XU and I. Howitt, Realistic energy model based energy balanced optimization for low rate WPAN network, Southeastcon, 2009. SOUTHEASTCON'09. IEEE. IEEE, 2009.

[20] J. Yang and Z. Fei, HDAR: Hole detection and adaptive geographic routing for ad hoc networks, Computer Communications and Networks (ICCCN), Proceedings of 19th International Conference. IEEE, 2010.
[21] Y. Gu, M. Pan and W. Li, Maximizing the Lifetime of Delay-Sensitive Sensor Networks via Joint Routing and Sleep Scheduling, 2014 International Conference on Computing, Networking and Communications(ICNC'14), Honolulu, Hawaii, USA.

[22] Y. Hao, M. Pan, Y. Fang and S. Glisic, Spectrum and Energy Efficient Relay Station Placement in Cognitive Radio Networks, IEEE Journal on Selected Areas in Communications (JSAC), Vol. 31, No. 5, pp. 883-893, May 2013.

[23] G. Joshi, On Relay-assisted Cellular Networks, Graduate thesis, India Institute of Technology-Bombay, 2010 


\section{Location Aided Energy Balancing Strategy in Green Cellular Networks}

\author{
Jianjun Yang \\ Department of Computer Science \\ University of North Georgia \\ Email: jianjun.yang@ung.edu
}

\author{
Zongming Fei \\ Lab for Advanced Networking \\ Department of Computer Science \\ University of Kentucky \\ Email: fei@netlab.uky.edu
}

\author{
Bryson Payne \\ Department of Computer Science \\ University of North Georgia, USA \\ Email: bryson.payne@ung.edu
}

Le $\mathrm{Li}$

David R.heriton School

of Computer Science

University of Waterloo, Canada

Email: 1248li@uwaterloo.ca

\author{
Markus Hitz \\ Department of Computer Science \\ University of North Georgia, USA \\ Email: markus.hitz@ung.edu
}

\author{
Tongquan Wei \\ School of Information Science \\ and Technology \\ East China Normal University, China \\ Email: tqwei@cs.ecnu.edu.cn
}

\begin{abstract}
Most cellular network communication strategies are focused on data traffic scenarios rather than energy balance and efficient utilization. Thus mobile users(cell phones) in hot cells may suffer from low throughput due to energy loading imbalance problem. In state-of-art cellular network technologies, relay stations extend cell coverage and enhance signal strength for mobile users. However, busy traffic makes the relay stations in hot area run out of energy quickly. In this paper, we propose an energy balancing strategy in which the mobile nodes are able to dynamically select and hand over to the relay station with the highest potential energy capacity to resume communication. Key to the strategy is that each relay station merely maintains two parameters that contains the trend of its previous energy consumption and then predicts its future quantity of energy, which is defined as the relay station's potential energy capacity. Then each mobile node can select the relay station with the highest potential energy capacity. Simulations demonstrate that our approach significantly increase the aggregate throughput and the average life time of relay stations in cellular network environment.
\end{abstract}

\section{INTRODUCTION}

During the past several years, there has been tremendous growth in cellular networks. With the introduction of Android and iPhone devices, use of ebook readers such as iPad and Kindle and the success of social networking giants such as Facebook, WeChat and QQ, the number of customers and the demand for cellular traffic have escalated significantly [25] [21] [26] [29]. The huge amount of customers and very high volume data transmission result in serious problems of energy consumption [1].

The rising energy costs of operating cellular networks have led to an emerging trend of addressing energy efficiency and energy balance utilization amongst the network operators and regulatory bodies such as 3GPP and ITU [2]. This trend has stimulated the interest of researchers in an innovative new research area called green cellular networks. In this regard, the European Commission has recently started new projects to address the energy issue of mobile communication systems, such as "Energy Aware Radio and Network Technologies (EARTH)", "Towards Real Energy-efficient Network Design" and "Cognitive Radio and Cooperative strategies for Power Saving in Multistandard Wireless Devices" [3]. Energy in cellular network is a vast research discipline that needs to cover all the layers of the protocol stack and various system architectures and it is important to identify the fundamental trade-offs linked with energy efficiency and the overall performance [4].

Scholars have addressed four key issues in terms of energy efficiency with network performance [2] [22] . They are deployment efficiency (balancing deployment cost, throughput), spectrum efficiency (balancing achievable rate), bandwidth (balancing the bandwidth utilized) and delay (balancing average end-to-end service delay). To address the challenge of increasing power efficiency in future cellular networks and thereby to maintain profitability, it is crucial to consider various paradigm-shifting technologies, such as energy efficient wireless architectures and protocols, efficient base station(BS) redesign, smart grids, opportunistic network access or cognitive radio, cooperative relaying and heterogeneous network deployment based on smaller cells.

In addition to academia, governments and industries have recently shown keen concerns on the critical issues related to energy efficiency and its balance utilization and in the ICT(Information and Communication Technology) area. However, as studied in the recent literature [5] [6], most of the techniques applied to current mobile networks have been designed by taking into account non-energy-related factors, such as throughput, Quality of Service (QoS), availability, scalability, and so on [33] [28] [34] [31] [20] [32] [27]. Moreover, in real-world systems, mobile users (MUs) 
are not evenly distributed across cells, resulting in that MUs in a hot cell will be affected by the load imbalance and they might unable to get services. Meanwhile, as novel network architectures that include picocells, hierarchical cells and femtocells emerge, the density of base stations and mobile users is becoming larger, and the cells go smaller. The appearance of this high density cellular networks introduces more variety of load across different cells and makes the load imbalance problem more serious [35] [36].

The new generation cellular networks allow mobile users to connect relay station and then connect to base station. The busy traffic near a relay station often makes the energy of the relay station go down very quickly. However, the relay station with low traffic is idle with high energy. In order to balance the energy utilization among different cells, it is needed to transfer the over-loaded traffic from hot cells to neighboring cooler ones. The challenge is how to balance the energy utilization of the relay stations in order to get the best trade-off among all the relay stations in a cellular network.

In this paper, we develop a statistical parameter based energy balance utilization algorithm, in which each relay station maintains the acceleration and the variance of energy consumption that represent the station's historical energy consumption acceleration and the variance. Together with its current energy quantity, the relay station is able to predict its future energy quantity, which is considered as its potential energy capacity. Before payload data transmission between a mobile user and a relay station, the mobile user disseminates a message to a portion of its neighbor relay stations depending on their geographic locations . Then it selects and hands over to the relay station with highest potential energy. Because each relay station only needs to maintain two parameters, the overhead of this scheme is very low. Our simulation results illustrate that our approach significantly increases the aggregate throughput in the network and the average life time of the relay stations compared with existing approaches.

The rest of the paper is organized as follows. Section II discusses the related research on this topic. Section III proposes a novel method that select the best relay station. We evaluate the proposed schemes by simulations and describe the performance results in Section IV. Section V concludes the paper.

\section{RELATED WORK}

Various energy utilization strategies in cellular networks were proposed and scholars developed various ways to solve energy utilization questions [37] [24] [38] [39]. The schemes are classified into several categories. The first one contains the strategies based on channel borrowing from cooler cells [9]. The second category includes the strategies based on BS selection [10]. The third category contains strategies based on power control and cell breathing [10] [11]. The last type consists of strategies on relay-assisted traffic transfer [12]. The basic idea of channel borrowing is to borrow a set of channels from cooler cells to hot cells. However, this will change the pre-defined spectrum reuse pattern and introduce more co-channel interference [7]. There are also a great deal of research combining multihop wireless network (MWN) with infrastructure wireless networks, forming architecture of multi-hop cellular network (MCN). In MCNs, relay stations (RSs) are network components that are dedicated to storing and forwarding data received from BSs to MUs, and vice versa. Deploying relay stations can extend the coverage and enhance the signal strength, which clearly help improve the performance for MUs near the edge of the cell. The appearance of MCNs also provides another method to solve the load imbalance among different cells. That is to transfer over-loaded traffic from hot cells to cooler cells by RSs. Compared with previous discussed dynamic load balancing schemes, relaybased load balancing schemes are more flexible and will introduce less interference. In [5], a mobile-assisted call admission scheme is proposed to achieve load balancing in cellular networks, which require an ad-hoc overlay network on the cellular network. The authors divided the channels into two parts, one for the ad-hoc overlay network, the other for the cellular network. In [6] [7], the authors proposed dynamic load balancing schemes in the integrated cellular and ad-hoc relaying systems (iCAR). The adhoc relaying stations (ARS) compose an overlay ad-hoc network, which can help relay traffic among different cells. As in iCAR systems, ARSs work on ISM-band channels, it is pointed out that the performance of iCAR systems will depend on the number of available ISM-band channels, and interference in ISM-band could affect the performance of dynamic load balancing.

A. Alam et al. [14] investigates dynamic traffic-aware BS switching modes, where the BS can alter its operating modes between standard BS operations and switching to relay station $(\mathrm{RS})$ mode, the so-called BS-RS Switching model. Depending on the traffic fluctuations, load profiles are divided into two categories. One is Zero-to-medium traffic period when a BS switches to the RS mode and turns off all its high-power consuming equipment. The second is Peak traffic period when all BS are fully active. The rationale for switching from a BS to RS mode is to ensure those MS that would be served by the switched off cell and may suffer deep fading, are still be able to receive the same QoS. Furthermore, since the propagation distance has been shortened between the MS and serving BS via the back haul connection, the required MS transmit power is concomitantly reduced compared with the BS sleep and cell zooming technique [5].

K. Xu and M. Zhou [15] [16] developed a realistic and representative energy model based on RF transceiver. As a typical IEEE 802.15.4 RF transceiver mode, each wireless node is equipped with multiple discrete transmit power levels. Based on this energy model, they developed a power control policy, which configure the transmit power level as a function of transmission distance such that the energy cost is minimized. Then, the optimized energy balanced 
chain model is proposed to determine the optimal traffic flow distribution.

\section{PROBLEM FORMULATION}

\section{A. The Basic Idea}

In cellular networks, each base station covers a number of cell phones in a hexagonal cell. We consider a cellular network containing a set of base stations(BS), relay stations(RS) and mobile users(cell phones)(MU), denoted by $\left\{B_{1}, B_{2}, \ldots, B_{s}\right\},\left\{R_{1}, R_{2}, \ldots, B_{t}\right\}$, and $\left\{m_{1}, m_{2}, \ldots, m_{p}\right\}$. Each BS is located at the center of the cell. Each RS is located at a boundary of cells. The traditional communication between two mobile users $m_{i}$ to $m_{j}$ is that $m_{i}$ communicates to its base station $B_{i}$, then $B_{i}$ communicates to $B_{j}$ which covers $m_{j}$, and then $B_{j}$ communicates to $m_{j}$. In new generation cellular network, the relay stations are used to assist communications between cell phone to cell phone or cell phone to base station. Fig. 11 shows an instance with several base stations, relay stations and cell phones, where the central cell is a hot cell with a large number of cell phones and busy data traffic and the other cells are cooler cells with less cell phones. Note that the cell phones in all figures of this paper represent active cell phones.

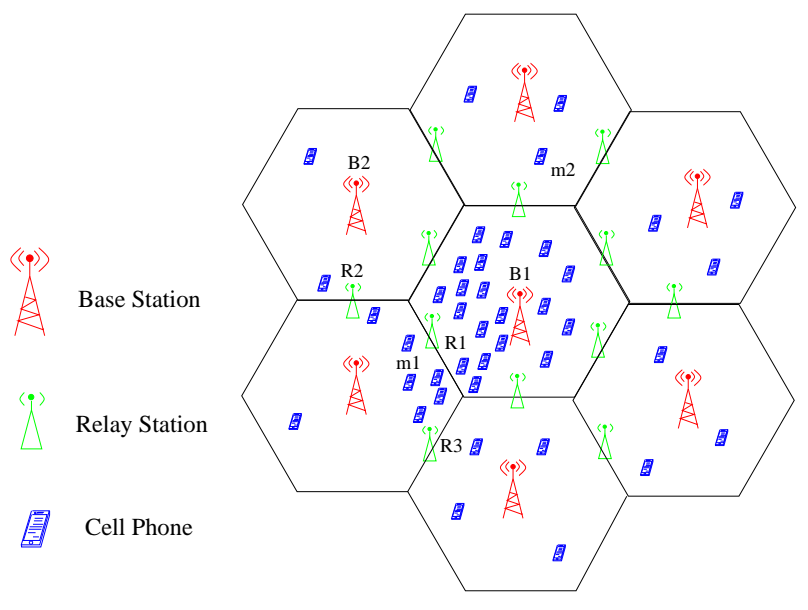

Fig. 1. A topology of a cellular network.

Data transmission takes place when a cell phone(initiator) intends to talk to another cellphone(receiver). The scenario is simple if the two are in the same cell [8]. We consider the circumstance that the receiver is in another cell. In Fig. 11 the communication between $m_{1}$ and $m_{2}$ is such an instance, in which data is relayed from $m_{1}$ to $R_{1}$, then from $R_{1}$ to $B_{1}$, and continues until it reaches $m_{2}$. In the scenario, $R_{1}$ is hot with a lot of cell phones nearby. Hence its energy is going down very quickly. However, $\mathrm{R}_{2}$ covers less cell phones and its energy is going down slowly. Here comes up with a question, can $m_{1}$ find $R_{2}$ or even $R_{3}$ to relay the message to bypass the hot relay station $R_{1}$ ?
Since a mobile cell phone is able to select a relay station and hand over to it [8], we develop an approach to let each mobile user find the RS with highest potential energy and hand over to it. We start our basic idea by a simplified case as Fig. 2. Suppose $m_{1}$ intends to talk to $m_{2}$. If $m_{1}$ selects $R_{1}$ to conduct relay, it may not be a good choice because $R_{1}$ is busy and may run out of energy shortly. $R_{1}$, $R_{2}$ and $R_{3}$ are all possible relay stations for $m_{1}$. Suppose the residual energy of $R_{1}$ is $900 \mathrm{kWh}, R_{2}$ is $800 \mathrm{kWh}$ and $R_{3}$ is $850 \mathrm{kWh}$ at current moment. We further assume that the historical residual energy vector up to this moment of $R_{1}$ is $[\ldots, 2000,1500,1300,900], R_{2}$ is $[\ldots, 900,870$, $830,800]$, and $R_{3}$ is $[\ldots, 1400,1200,1000,850]$. A simple strategy will let $m_{1}$ select the one that has the highest energy at the moment. Seemingly, $R_{1}$ is the best choice. However, since $R_{1}$ is in a hot area serving a large number of mobile users and its energy is going down dramatically, while $R_{2}$ are serving much less mobile users and its energy goes down slowly, $m_{1}$ should select $R_{2}$.

Our goal is to let $m_{1}$ select the relay station that will have the highest potential energy. In our approach, each RS can predict its future energy right after this time slot based on its historical and current energy, which is defined as potential energy of the RS in this paper. In this example, our strategy will let $m_{1}$ choose $R_{2}$.

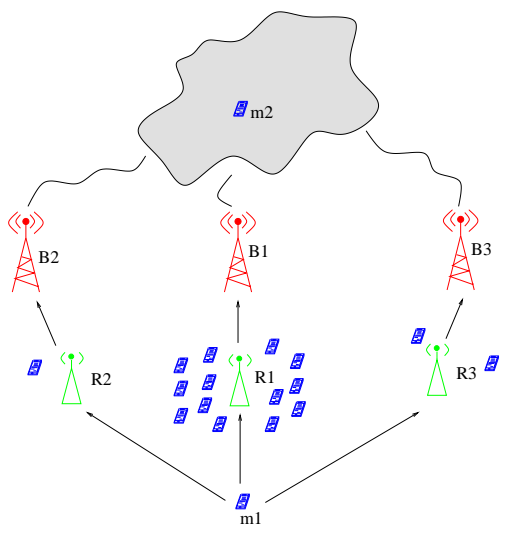

Fig. 2. The scenario to illustrate the main idea.

\section{B. Computation of Potential Energy}

The mobile initiator needs to know which relay station is the one that has the potential highest energy. The energy varying trend is essential to determine an RS's potential energy. Suppose the current time is $k$. A naive method on predicting the energy at time $k+1$ is as follows. Let $\mathrm{RS} \mathrm{R}_{i}$ save all its historical and current (time $k$ ) energy $E_{i_{0}}, E_{i_{1}}, \ldots, E_{i_{k}}$ in a vector. Then it can predict its energy for time $k+1$ by curve-fitting in numerical analysis that approximates its moving trend. The limitations of this method are to spend too much space to save the energy values in the vector and too complex for curvefitting computation. 
We propose a statistical based strategy to let each relay station predict its future energy. In this scheme, each RS only needs to maintain two parameters. One is its potential acceleration and the second is the variance of the acceleration. The two parameters store how its residual energy changes. Together with the RS's current energy, its future energy capacity can be calculated and is considered its potential energy. Thus, mobile user $m_{1}$ is able to figure out the best RS subsequently.

We define the following notations to represent the terms regarding our approach for node $R_{i}$.

$E_{i_{k}}$ : The measured energy at time $k$.

$\widehat{E_{i_{k}}}$ : The energy predicted at time $k$.

$a_{i_{k}}$ : The acceleration measured at time $k$. It indicates how energy changes during a time slot.

$\hat{a_{i_{k}}}$ : The acceleration at time $k$ evolved from time $k-1$.

$\mathrm{a}_{\mathrm{i}_{k}}$ : The potential acceleration at time $k$.

$v_{i_{k}}$ : The variance of acceleration updated at time $k$.

$v_{i_{k}}^{-}$: The variance of acceleration at time $k$ evolved from time $k-1$.

$\epsilon$ : The error or noise in the process.

$B_{i_{k}}$ : The blending factor at time $k$.

At time $k, R_{i}$ measures its energy $E_{i_{k}}$. And then it computes $a_{i_{k}}$ as its measured acceleration by

$$
a_{i_{k}}=\left(E_{i_{k}}-E_{i_{k-1}}\right) / \Delta t
$$

$R_{i}$ updates $\hat{a_{i_{k}}}$ and $v_{i_{k}}^{-}$in order to keep its historical energy to predict its future energy.

$$
\begin{gathered}
\hat{a_{i_{k}}^{-}}=a_{i_{k-1}} \\
v_{i_{k}}^{-}=v_{i_{k}-1}+\epsilon
\end{gathered}
$$

$R_{i}$ also computes the blending factor $B_{i_{k}}$, which indicates how much the acceleration changes from last time to current time.

$$
\mathrm{B}_{i_{k}}=v_{i_{k}}^{-}\left(v_{i_{k}}^{-}+\epsilon\right)^{-1}=v_{i_{k}}^{-} /\left(v_{i_{k}}^{-}+\epsilon\right)
$$

Once $R_{i}$ obtains the blending factor $B_{i_{k}}$ and the evolved acceleration $\hat{a}_{i_{k}}^{-}$, it knows how much the acceleration changes and the evolved acceleration. Additionally, $R_{i}$ considers the measured acceleration $a_{i_{k}}$. Then it calculates its potential acceleration $\hat{\alpha}_{\hat{i}_{k}}$. This acceleration will be used to predict its energy of time $k+1$.

$$
a_{i_{k}}=\hat{a_{i_{k}}}+B_{i_{k}}\left(a_{i_{k}}-\hat{a_{i_{k}}}\right)
$$

$R_{i}$ updates the variance of acceleration for future utilization.

$$
v_{i_{k}}=\left(1-B_{i_{k}}\right) v_{i_{k}}^{-}
$$

Finally, the energy predicted of time $k+1$ is

$$
E_{i_{k+1}}=E_{i_{k}}+a_{i_{k}} \Delta t
$$

At a certain time $k, R_{i}$ only needs to measure its energy $E_{i_{k}}$ and record two parameters $a_{i_{k-1}}^{\hat{}}$ and $v_{i_{k-1}}$. Then it can predict its energy at time $k+1$ by the calculation, which is the potential energy capacity of $R_{i}$.

\section{Algorithm Description}

In our approach, when a user $m_{1}$ intends to communicate to $m_{2}$, it selects the relay station with highest potential energy. Apparently, $m_{1}$ does not need to consider the relay stations in the opposite direction $m_{1} \rightarrow m_{2}$, such as $R_{4}$, $R_{5}$ and $R_{6}$ in Fig. 3 . Here $R_{1}, R_{2}$ and $R_{3}$ are candidates for $m_{1}$. How can $m_{1}$ know the three possible candidates among the six relay stations in the boundaries? In new generation cellular networks, each device is equipped with GPS and hence it knows its location. We assume that the initiator $m_{1}$ knows the location of the receiver $m_{2}$. The assumption is very common in geographic routing [17] [30] [23]. So $m_{1}$ finds out the three possible candidates in this way: It connects $m_{1}$ and $m_{2}$. The line segment intersects the hexagonal area at $p$. Then the relay station located at the edge where $p$ is located and the two relay stations located at its adjacent edges are considered as good relay candidates. In Fig. 3. $p$ is located at segment bc, so $R_{2}$ located at bc is considered as one of the candidates. The two adjacent edges of $b c$ are $a b$ and $c d$, so $R_{1}$ and $R_{3}$ are also considered as $\mathrm{m}_{1}$ 's candidates.

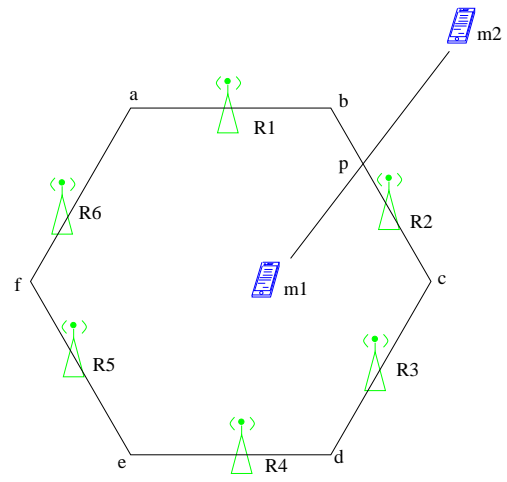

Fig. 3. To figure out three good relay stations.

In our strategy, when a cell phone(initiator) intends to talk to a receiver in a different cell, it calls the procedure Detection() to figure out the three possible relay stations by its location and the destination's location. In the procedure, it sends out a detection message to the three relay stations. Upon receiving the detection message, each RS calls Prediction() to calculate its potential energy and inform the energy capacity to the initiator. The initiator calls Selection() to find the relay station with possible highest potential energy, hands over to it and sends its payload to the RS.

\section{PERFORMANCE EVALUATION}

The performance evaluation was conducted in a simulated noiseless radio network environment using MATLAB. 


\section{Detection()}

Figure out the three candidate relay stations by its location and destination's location

Send detection message to the three relay stations

Fig. 4. Algorithm for initiator(MU) to send detection message

\section{Prediction()}

Calculate its potential energy by formula (7)

Send the energy quantity back to the initiator

Fig. 5. Algorithm of computation for potential energy

We create a topology that consists of 20 hexagonal cells. A base station is located in the center of each cell and a relay station is located at each edge. We randomly distribute a number of mobile users to the cells. We performed a sequence of experiments in which the number of mobile users is changed from 200 to 800 with increment of 50. For each number of mobile users, we measure the aggregate throughput 10 times and present the average. We also measure the average life time of the relay stations in our experiments. The life time of a relay station is important. If one RS runs out of energy and turns down, there will be a hole [13] and the whole throughput will be decreased dramatically.

Our approach considers energy balance. We compare our approach("EB by MU") with two other approaches. One is the scheme which considers energy balance but the balance is determined by base station("EB by BS"). The second one is without considering energy balance("No EB").

Fig. 7 shows our evaluation of aggregate throughput. Among the three approaches, the scheme without energy balance("No EB") has the lowest throughput. This indicates that energy balance can improve aggregate throughput because the traffic are allocated more reasonable to the relay stations. The throughput of our method is the highest one.

Fig. 8 shows the life time of the three schemes. The one without energy balance("No EB") results in the lowest average lift time. That is because when a relay station has busy traffic load, it runs of out energy quickly. And then there is a void or hole. The regular solution for hole problem is to find the landmark relay station [13]. However, the landmark has very heavy burden and it is exhausted quickly. This makes the network much worse. Our approach has the longest lift time because the communication between two

\author{
Selection() \\ stations \\ Compare the three energy quantities from the three relay \\ Select the RS with highest energy values \\ Hands over to the RS \\ Send payload to the RS
}

Fig. 6. Algorithm of hand over

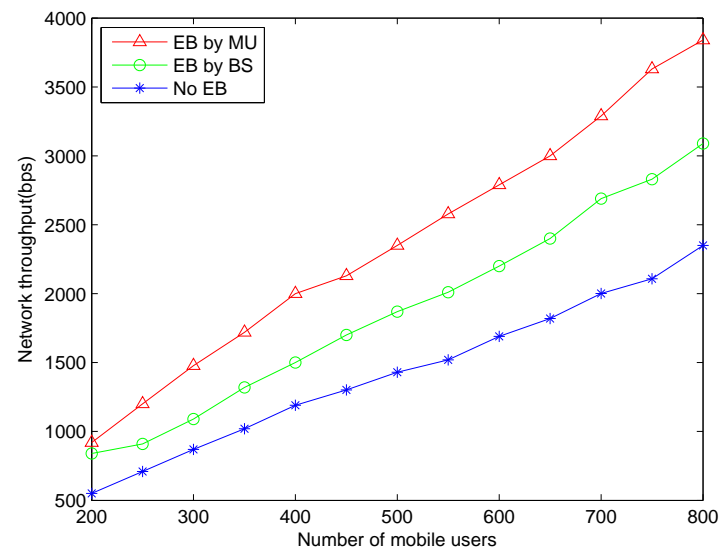

Fig. 7. Aggregate throughput

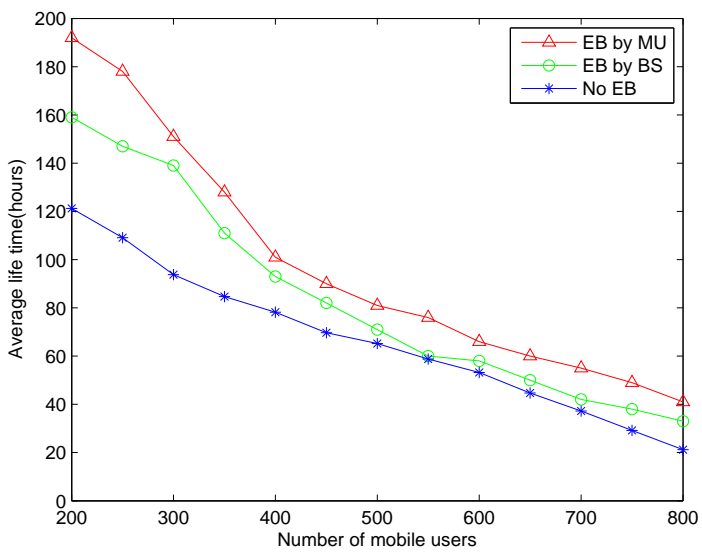

Fig. 8. Life time of relay stations

cell phones is always relayed by the RS with the highest potential energy. If a RS is busy and its energy goes down significantly, the new communication will not rely on it. Hence the balanced energy utilization makes the life time longer.

\section{CONCLUSION}

In this paper, we present a statistical based mechanism to balance energy utilization in cellular network. In the approach, each relay station only needs to remain two factor to store its historical energy trend and then it is able to predict its future energy. The message initiator selects the relay station with potential highest energy to relay data transmission. Simulations demonstrate that our approach results in higher throughput in the network and longer average life time of relay stations over related approaches. 


\section{REFERENCES}

[1] G. Fettweis and Z. Ernesto, ICT energy consumption-trends and challenges,Proceedings of the 11th International Symposium on Wireless Personal Multimedia Communications. Vol. 2. No. 4. 2008.

[2] H. Ziaul, H. Boostanimehr and V.K. Bhargava, Green cellular networks: A survey, some research issues and challenges, Communications Surveys \& Tutorials, IEEE 13.4 (2011): 524540

[3] M. Gruber, et al., EARTH: energy aware radio and network technologies,ersonal, Indoor and Mobile Radio Communications, 2009 IEEE 20th International Symposium on. IEEE, 2009.

[4] Y. Chen, et al., Fundamental trade-offs on green wireless networks,Communications Magazine, IEEE 49.6 (2011): 30-37.

[5] X. wang, et al., A survey of green mobile networks: Opportunities and challenges, obile Networks and Applications 17.1 (2012): 4-20.

[6] M. Etoh, T. Ohya and Y. Nakayama, Energy consumption issues on mobile network systems, Applications and the Internet, 2008. SAINT 2008. International Symposium on. IEEE, 2008.

[7] J. Yang and Z. Fei, Bipartite Graph Based Dynamic Spectrum Allocation for Wireless Mesh Networks, Distributed Computing Systems Workshops, 2008. ICDCS'08. 28th International Conference on (pp. 96-101). IEEE, Beijing, 2008.

[8] G. Josh, On Relay-assisted Cellular Networks, thesis, Department of Electrical Engineering, Indian Institute of Technology-Mombay, June, 2010

[9] S. Patra, et al., Improved genetic algorithm for channel allocation with channel borrowing in mobile computing,Mobile Computing, IEEE Transactions on 5.7 (2006): 884-892.

[10] S. Hanly, An algorithm for combined cell-site selection and power control to maximize cellular spread spectrum capacity,Selected Areas in Communications, IEEE Journal on 13.7 (1995): 1332-1340.

[11] M. Soleimanipour, W. Zhuang and G.H. Freeman, Optimal resource management in wireless multimedia wideband CDMA systems,SMobile Computing, IEEE Transactions on 1.2 (2002): 143160.

[12] E. Yanmaz, and O. Tonguz, Dynamic load balancing and sharing performance of integrated wireless networks, Selected Areas in Communications, IEEE Journal on 22.5 (2004): 862-872.

[13] J. Yang, and Z. Fei, ITGR: Intermediate Target Based Geographic Routing, Computer Communications and Networks (ICCCN), 2010 Proceedings of 19th International Conference on (pp. 1-6). IEEE, Zurich, Switzerland, August 2010.

[14] S. Alam, et al., Energy efficient relay-assisted cellular network model using base station Switching, Globecom Workshops (GC Wkshps), 2012 IEEE. IEEE, 2012.

[15] K. XU and M. Zhou, Energy balanced chain in IEEE 802.15. 4 low rate WPAN, Computing, Networking and Communications (ICNC), 2013 International Conference on. IEEE, 2013.

[16] K. XU and I. Howitt, Realistic energy model based energy balanced optimization for low rate WPAN network, Southeastcon, 2009. SOUTHEASTCON'09. IEEE. IEEE, 2009.

[17] J. Yang and Z. Fei, HDAR: Hole detection and adaptive geographic routing for ad hoc networks, Computer Communications and Networks (ICCCN), Proceedings of 19th International Conference. IEEE, 2010.

[18] G. Joshi, On Relay-assisted Cellular Networks, Graduate thesis, India Institute of Technology-Bombay, 2010

[19] Y. Su, Y. Wang, G. Agrawal and R. Kettimuthu, SDQuery DSI: integrating data management support with a wide area data transfer protoco,Proceedings of SC13: International Conference for High Performance Computing, Networking, Storage and Analysis, 2013.

[20] H. Zhang, Z.Zhang and H. Dai, On the Capacity Region of Cognitive Multiple Access over White Space Channels, Selected Areas in Communications, IEEE Journal on, Vol. 31, Number 11, Pages 2517-2527, (2013).
[21] J. Shen, P. Su and S. Cheung, Virtual Mirror Rendering with Stationary RGB-D Cameras and Stored 3D Background, IEEE Transactions on Image Processing, vol. 22, issue 9, pp. 1-16.

[22] K. Xu, D. Tipper, P. Krishnamurthy and Y. Qian, An Efficient Hybrid Model and Dynamic Performance Analysis for Multihop Wireless Networks, in Proceedings of 2013 IEEE International Conference on Computing, Networking and Communications (ICNC'13), San Diego, CA, USA, January 2013.

[23] J. Yang and Z. Fei, Broadcasting with Prediction and Selective Forwarding in Vehicular Networks, International Journal of Distributed Sensor Networks, 2013 (2013).

[24] L. Li, Evaluating Information Retrieval Systems With Multiple Non-Expert Assessors, (2013).

[25] J. Shen and S. Cheung Layer Depth Denoising and Completion for Structured-Light RGB-D Cameras, IEEE Conference on Computer Vision and Pattern Recognition (CVPR 2013), Portland, USA 2013.

[26] J. Shen and W. Tan, Image-based indoor place-finder using image to plane matching, IEEE International Conference on Multimedia and Expo (ICME 2013), San Jose, USA 2013.

[27] J. Shen et al., Automatic lip-synchronized video-self-modeling intervention for voice disorders, in e-Health Networking, Applications and Services (Healthcom), 2012 IEEE 14th International Conference on, 2012, pp. 244-249.

[28] Q. Sun, W. Yu, Q. Hao and F. Hu, A Multi-agent Based Intelligent Sensor and Actuator Network Design for Smart House and Home Automation,Journal of Sensor and Actuator Network. vol. 2, no. 3, pp. 557-588, 2013.

[29] J. Shen, A. Raghunathan, S. Cheung and R. Patel, Automatic Content Generation for Video Self Modeling, IEEE International Conference on Multimedia and Expo (ICME 2011), Barcelona, Spain 2011.

[30] J. Yang and Z. Fei, Statistical Filtering Based Broadcast Protocol for Vehicular Networks, 20th International Conference on Computer Communication Networks, Maui, Hawaii, USA, 2011.

[31] H. Zhang, Z. Zhang and H. Dai, Gossip-based Information Spreading in Mobile Networks, (2012).

[32] H. Zhang, Z. Zhang and H. Dai, Mobile Conductance in Sparse Networks and Mobility-Connectivity Tradeoff, arXiv preprint arXiv:1403.7691 (2014).

[33] Q. Sun, F. Hu and Q. Hao, Mobile Target Scenario Recognition via Low-cost Pyroelectric Sensing System: Towards a ContextEnhancement Accurate Identification,IEEE Transactions on Systems, Man and Cybernetics: Systems. pp. 2168-2216, 2013.

[34] Q. Sun, R. Ma, F. Hu and Q. Hao, Space Encoding Based Human Activity Modeling and Situation Perception,In Proceeding of 2013 IEEE International Multi-Disciplinary Conference on Cognitive Methods in Situation Awareness and Decision Support, pp. 186-189, 2013.

[35] Y. Wang, W. Jiang and G. Agrawal, SciMATE: A Novel MapReduceLike Framework for Multiple Scientific Data Formats,Cluster, Cloud and Grid Computing (CCGrid), 2012 12th IEEE/ACM International Symposium on, PP 443-450, 2012.

[36] Y. Wang, Y. Su and G. Agrawal, Supporting a Light-Weight Data Management Layer over HDF5,Cluster, Cloud and Grid Computing (CCGrid), 2013 13th IEEE/ACM International Symposium on, PP 335-342, 2013.

[37] L. Li and M. Smucker, Tolerance of Effectiveness Measures to Relevance Judging Errors, European conference on Information Retrieval, 2014.

[38] Y. Zhou, L. Li and H. Zhang, Adaptive Learning of Regionbased pLSA Model for Total Scene Annotation, ArXiv preprint arXiv:1311.5590 (2013).

[39] Y. Zhou, L. Li, T. Zhao and H. Zhang, Region-based high-level semantics extraction with CEDD, Network Infrastructure and Digital Content, 2010 2nd IEEE International Conference on. IEEE, 2010. 